\title{
Measured sucrose preference in the albino rat: The influence of an auditory cue'
}

\section{Abstraet}

Data is presented to support a contention that the measured preferences for sapid solutions may be enhanced by association with non-gustatory cues. Albino rats were given a two-bottle preference test for various concentrations of sucrose compared with distilled water. The measured preference for sucrose at lower concentrations was increased when white noise was paired with the sucrose.

\section{Problem}

Previous preference work (Fisher, 1964) has suggested that the measured preference for .1 M NaCl declines when positional and visual cues are removed from a successive two-bottle preference test. This study deals with the possibility that measured preference for sucrose can be increased by the addition of a known non-taste cue to a previously taste-dependent situation.

\section{Method}

The subjects were 19 male albino rats of Charles River stock circa $250 \mathrm{gm}$ at the beginning of testing. The apparatus consisted of a small plexiglass chamber with a single aperture located on one wall. Drinking tubes containing sucrose and distilled water were presented alternately for 1-min. intervals thru this aperture. Food was always available in a box fastened to the wall opposite the aperture. Records of licking were obtained using an electronic drinkometer, counters and a cumulative response recorder; food-box entries were recorded on the same records. The animals were placed in the testing box for $96 \mathrm{hr}$. All sucrose solutions were made with reagent grade sucrose and distilled water. They were made every $24 \mathrm{hr}$. and allowed to come to room temperature before presentation to the animals. A small loudspeaker, connected to a white-noise source, was located directly above the testing chamber. White noise $(85 \mathrm{db})$ was paired with the $1-\mathrm{min}$. presentations of sucrose for 12 animais and on alternate minutes for the two animals run to water paired with water. Table 1 illustrates the testing scheme.

\section{Results}

Figure 1 illustrates measured preferences, for all rats, by molar concentration. The ordinate shows the proportion of total lapping applied to the sucrose tube. Where water was paired with water-plus-noise, the ordinate represents the proportion of total lapping applied to the tube whose presentation was accompanied by the white noise. The horizontal dashed line at the .50 level indicates where the plotted data would fall if the licking were equally distributed between the two tubes.
Table 1

No Noise Cue

Noise Cue

\begin{tabular}{|c|c|c|c|}
\hline 3 Rats & $\begin{array}{l}.3 \text { M Sucrose } \\
\text { vs. Water }\end{array}$ & & \\
\hline \multirow[t]{7}{*}{2 Rats } & $\begin{array}{l}\text {.1 M Sucrose } \\
\text { vs. Water }\end{array}$ & 2 Rats & $\begin{array}{l}.1 \text { M Sucrose } \\
\text { vs. Water }\end{array}$ \\
\hline & & 2 Rats & $\begin{array}{l}.09 \text { M Sucrose } \\
\text { vs. Water }\end{array}$ \\
\hline & & 2 Rats & $\begin{array}{l}.07 \mathrm{M} \text { Sucrose } \\
\text { vs. Water }\end{array}$ \\
\hline & & 2 Rats & $\begin{array}{l}.05 \text { M Sucrose } \\
\text { vs. Water }\end{array}$ \\
\hline & & 2 Rats & $\begin{array}{l}.03 \mathrm{M} \text { Sucrose } \\
\text { vs. Water }\end{array}$ \\
\hline & & 2 Rats & $\begin{array}{l}.01 \text { M Sucrose } \\
\text { vs. Water }\end{array}$ \\
\hline & & 2 Rats & $\begin{array}{l}\text { Water with } \\
\text { noise } \\
\text { vs. Water }\end{array}$ \\
\hline
\end{tabular}

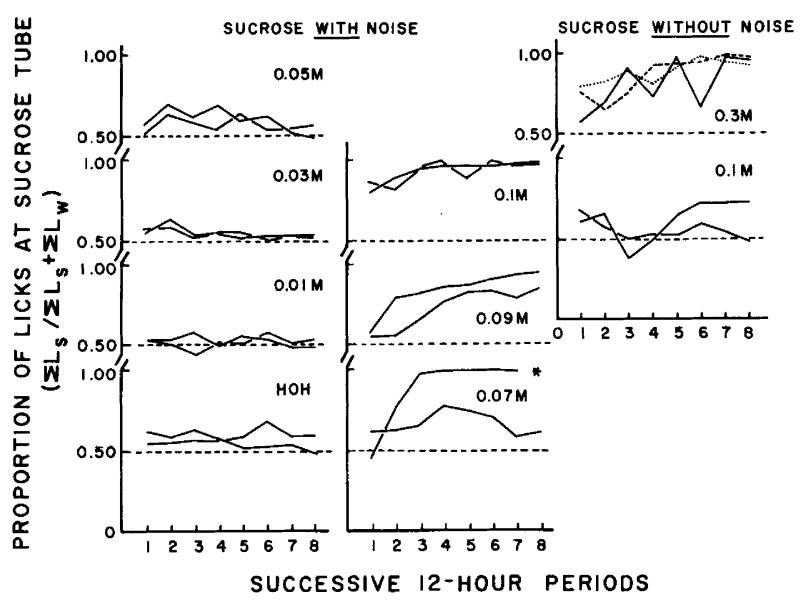

Fig. 1. Proportion of total licking at the sucrose tube for all animals, over 8 successive 12 -hour periods is shown. Numerals indicate the molar concentration of the sucrose solution. HOH, two water tubes presented, one accompanied by white noise. Left side of the figure illustrates licking when white noise accompanied presentation of the sucrose tube; right side, no accompanying noise. $\Sigma L_{S}$, total licks at sucrose tube over 12 hours, $\Sigma L_{W}$, total number or licks at water tube over 12-hour period. $*$, apparatus breakdown; data not recorded. 
The most notable result is a comparison between the rats licking at .1 M Sucrose paired with water and those licking at .1 M Sucrose-with-noise paired with water. Rats in the former case showed an almost immediate preference for the sucrose that reached a high value by the $36 \mathrm{th} \mathrm{hr}$. of testing. Rats run to $.1 \mathrm{M}$ Sucrose and water displayed a slight initial preference for sucrose that declined quickly and in one case improved after the $48 \mathrm{th} \mathrm{hr}$. of testing. Another interesting point is the gradual development of the measured preference for . 3 M Sucrose over the first $72 \mathrm{hr}$. The rats tested on $.1 \mathrm{M}$ Sucrose in the noise-cue condition developed their preferences more rapidly than did the $.3 \mathrm{M}$ Sucrose animals without a noise-cue. The first sign of a stable preference for sucrose occurred at .07 $\mathrm{M}$ concentration. One animal shows a stable preference by the 36 th $\mathrm{hr}$. The other animal begins to decline in sucrose-preference after the 48 th $\mathrm{hr}$.

The cumulative records that monitored all intake showed that most, though not all, eating occurred just prior- and subsequent-to eating. Periods of self-deprivation ranged from 1-6 hr. Most self-deprivation ranged between 1 and $3 \mathrm{hr}$. Continued licking lasted for as little as a few seconds and in some cases continued for half an hour. Most licking ran for 10-15 min. stretches.

\section{Diseussion}

The data may be interpreted to show that preference functions derived from various testing techniques may be non-linear with respect to the animal's stimulus bias. The sapid stimuluation may be considered reinforcement that both generates and sustains instrumental responding. A testing situation with an abundance of cues associated with reinforcement of an instrumental response may show a learning curve similar to that shown by the three rats run to $.3 \mathrm{M}$ Sucrose (no noise cue) and water. If an average is taken for $48 \mathrm{hr}$. responding as an indication of preference, the figure will be at a lower level than the terminal preference. A testing situation with an abundance of cues may cause the data to reveal lower thresholds than one with minimal extra-gustatory information. The importance of non-gustatory cues appears to vary inversely with the strength of the stimulus, e. g., .1 M Sucrose with and without noise, $.3 \mathrm{M}$ Sucrose without noise in Figure 1. The 1-min. between sucrose presentation did not appear to function as an effective temporal cue. Falk (1963) found that preference functions for saline preference increased as time-of-presentation increased from $5 \mathrm{~min}$. to 10 min. to $15 \mathrm{~min}$. in a successive preference method. The function was nearly horizontal at $5 \mathrm{~min}$. suggesting that our 1-min. presentation precluded temporal discrimination by the rats.

\section{Referenees}

FALK, J. L., \& TITLEBAUM, L. F. Saline solution preference in the rat: further demonstrations. J. comp, physiol. Psychol., 1963, 56, 337-342.

FISHER, G. L. Measured saline preference in the albino rat: the role of nongustatory factors. Psychon. Sci., 1964, 1, 45-46.

\section{Notes}

1. This research was conducted under grant G-14332 from the National Science Foundation.

2. The author is indebted to Miss Judith Jay for collecting a large portion of the data reported in this paper.

\section{Errata}

The Editor regrets that two errors appeared in the November issue of the journal. In the first case (Ashida), two capital sigmas were omitted in a formula. In the second case (Cross), a line was omitted, and the omitted material is indicated below by underlining.

ASHIDA, SACHIO. Modification of early experience of the tendency toward gregariousness in rats. Psychon. Sci., 1964, 1, 343-344.

$$
\mathrm{n}
$$

$$
\begin{aligned}
& 1 / n \cdot \sum_{1} \log f\left(T_{j g I}\right)= \\
& 1 / n \cdot \sum_{i}^{n} \log m_{j}\left(R_{E I}\right)-1 / n \cdot \sum_{i}^{n} \log m_{j}\left(R_{C i}\right)
\end{aligned}
$$

CROSS, HENRY A. Reply (to Routtenberg's Comment concerning Cross, Rankin, \& Wilson). Psychon. Sci., $1964,1,334$.

This was clearly a call for research, and the two factors discussed by Routtenberg provide a perfectly acceptable starting place. However, Routtenberg's proposed research would not necessarily control for the visual factor since it remains a distinct possibility that operation-induced emotionality or disruption might be quite different for two organisms which differed greatly in their initial dependence. 